\title{
Quasispecies theory in the context of population genetics Claus O Wilke*1,2
}

Address: ${ }^{1}$ Keck Graduate Institute of Applied Life Sciences, 535 WatsonDrive, Claremont, California 91711, USA and ${ }^{2}$ Digital Life Laboratory, California Institute of Technology, Mail Code 136-93, Pasadena, California 91125, USA

Email: Claus O Wilke* - wilke@kgi.edu

* Corresponding author

Published: 17 August 2005

BMC Evolutionary Biology 2005, 5:44 doi:10.1 186/147|-2/48-5-44

This article is available from: http://www.biomedcentral.com/I47I-2/48/5/44

(c) 2005 Wilke; licensee BioMed Central Ltd.

This is an Open Access article distributed under the terms of the Creative Commons Attribution License (http://creativecommons.org/licenses/by/2.0), which permits unrestricted use, distribution, and reproduction in any medium, provided the original work is properly cited.
Received: 16 February 2005

Accepted: 17 August 2005

\begin{abstract}
Background: A number of recent papers have cast doubt on the applicability of the quasispecies concept to virus evolution, and have argued that population genetics is a more appropriate framework to describe virus evolution than quasispecies theory.

Results: I review the pertinent literature, and demonstrate for a number of cases that the quasispecies concept is equivalent to the concept of mutation-selection balance developed in population genetics, and that there is no disagreement between the population genetics of haploid, asexually-replicating organisms and quasispecies theory.

Conclusion: Since quasispecies theory and mutation-selection balance are two sides of the same medal, the discussion about which is more appropriate to describe virus evolution is moot. In future work on virus evolution, we would do good to focus on the important questions, such as whether we can develop accurate, quantitative models of virus evolution, and to leave aside discussions about the relative merits of perfectly equivalent concepts.
\end{abstract}

\section{Background}

Quasispecies theory describes the evolution of an infinite population of asexual replicators at high mutation rate $[1,2]$. Quasispecies theory is often cited as the theory to describe the evolution of RNA viruses [3], but in recent years several authors have questioned whether quasispecies theory has any relevance for virus evolution [4-7]. Esteban Domingo has responded to this criticism from an experimentalist's point of view [8]. However, the fundamental issue in this discussion is of theoretical nature, and has not yet been addressed in detail. The fundamental issue is whether quasispecies theory and population genetics are two competing theories, and whether virology ulitmately has to decide for or against one or the other. Some quasispecies opponents have argued that quasispecies theory contradicts population genetics (e.g. "This model contrasts sharply with conventional population genetics models ..." in [7]), and that there is no evidence that favors quasispecies theory over classical population genetics [6]. On the other hand, some quasispecies proponents have also voiced the position that quasispecies theory goes beyond population genetics, and virologists in general have frequently used the term quasispecies inappropriately (see e.g. the discussion on this topic by Eigen, Ref. [9]). I find this discussion somewhat frustrating, because quasispecies theory is simply a subset of theoretical population genetics, and it is mathematically equivalent to the theory of mutation-selection balance. The only real difference between quasispecies theory and mutationselection balance is that they have been developed largely independently by two separate schools of research, and that these schools of research have often focused on 
somewhat different questions and special cases. Quasispecies theory treats multiple loci, whereas early work on mutation-selection balance has focused on one- or twolocus models. On the other hand, most work on population genetics considers finite populations and includes stochastic effects, whereas quasispecies theory is first and foremost a deterministic description of infinite populations.

Quasispecies theory has its origin in a seminal paper written by Eigen in 1971 [10], in which he studied the errorprone self-replication of biological macromolecules, primarily with the goal of understanding the origin of life. However, Eigen did not yet use the term quasispecies in this 1971 paper; he coined this term in a later paper coauthored with Peter Schuster [11]. These early papers by Eigen, Schuster, and coworkers (reviewed in [1,2]) were some of the first to study theoretically the extreme nucleotide heterogeneity caused by highly error-prone replication. As a consequence, they generated interest among researchers working on RNA viruses, as these viruses were found to replicate at high mutation rates and have extremely polymorphic populations [3,12-14].

Eigen's papers also generated substantial interest among theoreticians (mostly physicists), who found the description of highly error-prone replication an interesting theoretical challenge. Unfortunately, much of the theoretical follow-up work [15-21] has focused on a particular fitness landscape, the single-peak (or master sequence) fitness landscape, in which a single sequence (the master sequence) has superior fitness $1+s$, and all other sequences have inferior fitness 1 . As a result, much of the generality of Eigen's original work, as well as its connection to population genetics, have been obscured, and the conclusions of these special-case studies are frequently taken to be general predictions of quasispecies theory.

Because of the development of quasispecies theory independently from population genetics, and because of the widespread emphasis on a single fitness landscape in quasispecies theory, many authors now hold a set of beliefs about quasispecies theory that do not correspond to the actual predictions of the theory. These beliefs are:

1. Quasispecies theory is at odds with population genetics.

2. Quasispecies theory is inapplicable if populations are finite and there is neutral drift.

3. Quasispecies theory predicts an error threshold.
In the next three sections, I will address each of these points in detail. However, first I have to define what exactly I mean by quasispecies theory.

Throughout this paper, by quasispecies theory I mean specifically Eq. (6) in Ref. [11],

$$
\dot{x}_{i}(t)=\left[W_{i i}-E(t)\right] x_{i}(t)+\sum_{j \neq i} W_{i j} x_{j}(t)
$$

where $x_{i}(t)$ is the concentration of sequence $i, W_{i j}=A_{j} Q_{i j}$ is the product of the replication rate (fitness) $A_{j}$ of sequence $j$ and the mutation probability $Q_{i j}$ from sequence $j$ to $i$, and $E(t)$ is the total production of new sequences,

$$
E(t)=\sum_{k} \sum_{j} W_{k j} x_{j}(t)
$$

In my definition of quasispecies theory, I also include straightforward generalizations of the above equation that have been used in the quasispecies literature, such as the discrete-time quasispecies equation, which can be written as $[22,23]$

$x_{i}(t+1)=\sum_{j} W_{i j} x_{j}(t) / E(t)$

and leads to the same steady-state solution as Eq. (1). Both Eqs. (1) and (3) can be mapped onto linear equations, and then solved by diagonalizing the matrix $W_{i j}$. In both cases, the steady-state solution is given by the dominant eigenvector of $W_{i j}$.

The mapping onto a linear system assumes that the $W_{i j}$ which consist of the fitness landscape (as given by the $A_{j}$ ) and the mutation landscape (as given by the $Q_{i j}$ ), are constants. In the most general case, fitness will depend on the mutant frequencies $x_{i}$, as different mutants may make use of different resources, and the relative resource concentrations change as the mutant frequencies change. It turns out that the mapping onto a linear system is still valid if resource abundances change due to external factors [24], but not if resources change in response to increasing or decreasing mutant frequencies $x_{i}$. In this latter case, which corresponds to frequency-dependent selection, the conclusions drawn from quasispecies theory do not apply.

\section{Is quasispecies theory at odds with population genetics?}

Several recent papers present quasispecies theory as a theory that is alternative to (and maybe even contradictory to) standard population genetics [6,7]. Is there any merit to this position? Is quasispecies theory somehow at odds with standard population genetics? 
Let us investigate what form the quasispecies equations take in a simple example. Consider a single locus with two alleles a and A, and assume that the A allele has a selective advantage $s$ over the a allele. Further, assume that allele a mutates into allele $\mathrm{A}$, and likewise allele $\mathrm{A}$ into allele $\mathrm{a}$, with probability $\mu$. Then, in Eq. (1), we have $W_{\mathrm{AA}}=(1+$ $s)(1-\mu), W_{\mathrm{aA}}=(1+s) \mu, W_{\mathrm{Aa}}=\mu, W_{\mathrm{aa}}=1-\mu$, and hence (note that $x_{\mathrm{a}}(t)=1-x_{\mathrm{A}}(t)$ )

$$
\begin{aligned}
\dot{x}_{\mathrm{A}}(t) & =\left[(1+s)(1-\mu)-s x_{\mathrm{A}}(t)-1\right] x_{\mathrm{A}}(t)+\mu\left[1-x_{\mathrm{A}}(t)\right] \\
& =s x_{\mathrm{A}}(t)\left[1-\mu-x_{\mathrm{A}}(t)\right]+\mu\left[1-2 x_{\mathrm{A}}(t)\right] .
\end{aligned}
$$

If we set the mutation rate $\mu$ to zero, then this equation turns into

$\dot{x}_{\mathrm{A}}(t)=s x_{\mathrm{A}}(t)\left[1-x_{\mathrm{A}}(t)\right]$,

that is, into the standard logistic equation that describes the rise of a beneficial allele in an otherwise homogeneous population. Thus, we can recover standard population dynamics from the quasispecies equations. Now, let us calculate the steady state solution of Eq. (4) for an arbitrary mutation rate. We set $\dot{x}_{\mathrm{A}}(t)=0$, and find

$x_{\mathrm{A}}=\frac{1}{2}\left[1-\mu-2 \mu / s+\sqrt{(1-\mu-2 \mu / s)^{2}+4 \mu / s}\right]$,

and of course $x_{\mathrm{a}}=1-x_{\mathrm{A}}$. For $\mu=0$, this expression becomes $x_{\mathrm{A}}=1$, which simply means that the A allele will reach fixation in the absence of any mutation pressure. As $\mu$ increases, $x_{\mathrm{A}}$ decreases, and $x_{\mathrm{a}}$ increases. For a positive $\mu$, even though the a allele is removed from the population by selection, it is constantly regenerated from the A allele by mutation pressure, and thus reaches a positive equilibrium frequency. If the mutation rate is sufficiently high, then the equilibrium frequency of the a allele, maintained by the balance of selection and mutation pressure, can be substantial. In summary, we find that for the case of a single locus with two alleles, the quasispecies model predicts logistic growth of the beneficial allele in the absence of mutations, and mutation-selection balance in the presence of mutations.

Now consider the multi-locus case. A classic paper on mutation-selection balance is the one by Kimura and Maruyama, written in 1966 [25]. In this paper, Kimura and Maruyama study the mutational load of a haploid, asexually reproducing population. I will now show that this model is also a special case of the quasispecies equations. Kimura and Maruyama assume that the frequency $x_{i}$ of a sequence with $i$ mutations changes from one generation to the next according to (Eq. (3.1) in Ref. [25]):

$x_{i}^{\prime}=\sum_{j=0}^{i} \frac{w_{i-j} x_{i-j}}{\bar{w}} \frac{\mu^{j}}{j !} e^{-\mu}$, where $w_{i}$ is the fitness of a sequence with $i$ mutations, $\bar{w}=\sum_{i=0}^{\infty} w_{i} x_{i}$, and $\mu$ is the mutation rate (note that Kimura and Maruyama use $f_{i}$ instead of $x_{i}$ and $2 M$ instead of $\mu$ ). Now, define the mutation matrix $Q_{i j}$ as

$Q_{i j}= \begin{cases}\mu^{i-j} e^{-\mu} /(i-j) ! & \text { for } i \geq j, \\ 0 & \text { for } i<j,\end{cases}$

and write the matrix $W_{i j}$ in Eq. (3) as $W_{i j}=w_{j} Q_{i j}$. Then, we see that $E(t)$ as defined in Eq. (2) becomes $E(t)=\sum_{j=0}^{\infty} w_{j} x_{j}=\bar{w}$. Furthermore, the sum $\sum_{j} W_{i j} x_{j}$ in Eq. (3) runs from $j=0$ to $j=i$, since $Q_{i j}=0$ for $i<j$. After introducing a new index $k=i-j$, we can rewrite the sum as

$$
\begin{aligned}
\sum_{j=0}^{i} W_{i j} x_{j} & =\sum_{k=0}^{i} W_{i i-k} x_{i-k} \\
& =\sum_{k=0}^{i} w_{i-k} x_{i-k} \frac{\mu^{k}}{k !} e^{-\mu},
\end{aligned}
$$

which demonstrates that Eq. (7) follows directly from the quasispecies equation Eq. (3). As a consequence, the quasispecies model is in agreement with the Haldane-Muller principle [26], which means that the mutational load $L$ of a population described by the quasispecies model is in many (but not all) cases approximately given by $L=1-e^{-}$ $\mu$. (Deviations from this principle arise for example from the presence of neutral mutations $[27,28]$.)

Now that we have seen that quasispecies theory and mutation-selection balance are equivalent, the question remains whether Eigen just reinvented parts of population genetics, or actually contributed to the development of the field. While Eigen was not the first to consider mutation-selection balance (this concept goes back to Wright and Fisher in the early 20th century), by studying multilocus mutation-selection equations at arbitrary mutation rate he was certainly at the forefront of theoretical population genetics in the late 1970 s and early 1980s. The first analytic solutions to equations of the form Eq. (1) were found by Thompson and McBride in 1974 [29] and independently by Jones et al. in 1976 [30]. These works were directly influenced by Eigen's seminal paper of 1971 [10]. On the population genetics side, Moran was the first to solve Eq. (3) [31], also in 1976, but was unaware of the work by Eigen, Thompson, McBride, Jones, and coworkers.

One of the reasons why the quasispecies model is sometimes perceived to be at odds with standard population genetics is that it predicts (under certain conditions, I should add) that the equilibrium state of the population, which is given by the dominant eigenvector of the matrix 

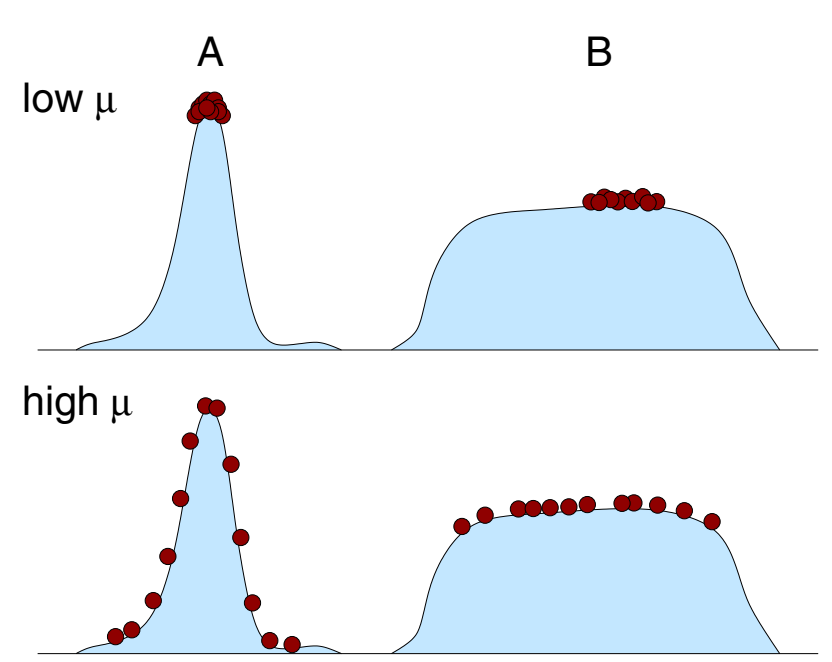

Figure I

Schematic drawing of the survival-of-the-flattest effect. At low mutation rate $\mu$, all individuals accumulate close to the top of the local fitness peak, and hence the individuals on peak A outcompete the individuals on peak B. At high mutation rate, most individuals on the steep peak $A$ are located at low fitness values, while the individuals on the flat peak $B$ remain close to the local optimum. As a consequence, the mean fitness of the individuals on peak $B$ exceeds that of the individuals on peak $A$, and thus the former outcompete the latter.

$W_{i j}$, is a stable mixture of closely related mutants. This mixture of mutants, also called a mutant cloud or quasispecies, does not necessarily have to contain the fastest-replicating individual sequence that exists in the fitness landscape. In other words, sequences with high fitness can lose out against sequences with lower fitness that have better support from their mutational neighbors [32,33], an effect which has been termed survival of the flattest (Figure 1).

It is important to understand that the emergence of a quasispecies is not something that has been put into the model ad hoc, but is a necessary consequence of the mutation-selection equations. We see in Eqs. (1) and (3) that the model is built on reproduction of individual sequences, but that mutations provide coupling between the different sequence types. When the mutation rate is low, then the quasispecies model predicts that the fastestreplicating sequence takes over the population, as we witness from the emergence of the logistic equation Eq. (5) at zero mutation rate. However, when the mutation rate is high, then the coupling between sequences caused by mutations can become stronger than the individual selection coefficients, and a quasispecies forms. Note that this effect will arise in any model of mutation-selection balance that correctly takes into account the coupling of different mutants at high mutation rate.

\section{Does quasispecies theory apply to finite populations?}

In the previous section, I have established that the quasispecies model is equivalent to the theory of mutationselection balance in an infinite, haploid, asexual population. However, this equivalence does not necessarily imply that the quasispecies model applies to populations of RNA viruses, because these populations are finite. Jenkins et al. [5] argue that the total sequence space of an RNA virus is much larger than the sequence space a finite population of realistic size can cover, and that therefore the deterministic equations of the quasispecies model are inapplicable, because virus evolution is dominated by random genetic drift. A priori, this is a reasonable objection, and we have to test whether the quasispecies equations are indeed useless in any realistic setting, or whether maybe complete coverage of the sequence space is not necessary to observe quasispecies effects. (By quasispecies effects, I mean that the population behaves in a way that can only be explained through strong mutational coupling between genotypes. An example would be the observation of the survival of the flattest effect.)

First, let us have a look at some theoretical studies of finite populations that have been carried out within the context of the quasispecies model [19,34-38]. In general, in these studies the deterministic equations are taken as the starting point, and then the authors derive corrections to these equations that take into account deviations from the deterministic behavior caused by the finite population size. Thus, at least in these model systems, the deterministic equations provide a reasonable starting point to understand the population dynamics. Van Nimwegen et al. make this point particularly clear by showing that in certain cases, we can understand the behavior of a finite population from a deterministic description of a population that occupies the sequence space around a local optimum [37]. In this model, information about other local optima (which would be available to an infinite population) is not necessary to accurately describe the behavior of the finite population on the given local optimum.

However, one could still object that these models may be describing idealized and simplified situations that differ too much from the reality of RNA viruses to be of any relevance. To counter this argument, we have to ask whether there is a more general way to determine the relevance of quasispecies theory to finite populations of RNA viruses. The hallmark of quasispecies theory (and of course of any theory of mutation-selection balance) is that for a sufficiently high mutation rate, we must take into account the 
formation of a quasispecies to obtain a faithful description of the population dynamics. Therefore, the question is under what conditions does mutational coupling become so strong that we can observe quasispecies effects in a finite population. Can theory help us to address this question?

In the formation of a quasispecies, the population minimizes the mutational load by accumulating sequences that have a reduced probability to suffer from deleterious mutations $[27,28,39,40]$. This effect has been termed evolution of mutational robustness [27]. Van Nimwegen et al. studied this effect for RNA evolution, and found that mutational robustness evolved as long as the product of mutation rate $\mu$ and effective population size $N_{e}$ was significantly larger than one, $\mu N_{e} \gg 1$ [27]. I have recently made similar observations in simulations of protein evolution [41]. What is interesting about the latter simulations is that in the regime of mutational robustness, the proteins continued to accumulate mutations, and in fact accumulated mutations faster than in the regime in which mutational robustness did not evolve. This observation demonstrates that the existence of a stable master sequence is not a necessary consequence of quasispecies evolution, in contrast to the key assumption of the study by Jenkins et al. [5]. These results can be understood with the theory of quasispecies fixation, which shows that an individual invading sequence has a positive fixation probability precisely when the mutant cloud that this sequence will spawn has higher fitness than the currently established mutant cloud, regardless of the individual fitness of the invading sequence $[42,43]$. Note that, in line with the previous section, this theory is again equivalent to the general theory of fixation in a haploid, asexually replicating population $[44,45]$.

Finally, the recent paper by Comas et al. [7] also provides evidence that quasispecies effects can be observed in surprisingly small populations, populations far too small to cover the relevant sequence space. Comas et al. studied to what extent the survival-of-the-flattest effect would be affected by population size, and found that population size played only a minor role in determining the position of the critical mutation rate at which the flatter strain began to outcompete the fitter strain. (I had previously found similar results in simulated RNA evolution [46].) Even in populations of size 250, Comas et al. consistently observed outcompetition of the fitter strain by the flatter strain at high mutation rate. Note that the digital organisms in these experiments had sizes of between 54 and 272 instructions, chosen from an alphabet of 28 different instructions, so that the complete sequence space of these organisms was between $10^{47}$ and $10^{68}$ sequences large. Clearly, a population of size 250 (or even several thou- sand, for that matter) cannot even come close to complete coverage of such a huge sequence space.

The previous paragraphs show that on purely theoretical grounds, there is no reason to assume that quasispecies effects cannot play a role in finite populations of RNA viruses. Nevertheless, to date we have no experimental evidence that unequivocally demonstrates such effects in a specific experimental system. On the other hand, selection for specific, individual mutants is common (see e.g. Ref. [47]). What does this experimental evidence imply for quasispecies theory? First, quasispecies theory covers both cases, those in which mutational coupling can be neglected, and those in which it can't. The latter is a second-order effect that becomes relevant only when there is no strong selection for individual sequences [40]. Thus, it is not surprising that in cases where selection is strong, such as in the case of resistance or escape mutants, we don't see quasispecies effects. Second, because quasispecies effects are second-order, it may be difficult to detect them experimentally, and experiments more sensitive than the ones carried out to date may be necessary to demonstrate their presence unequivocally.

In summary, we currently have no evidence (theoretical or experimental) that contradicts the existence of quasispecies effects in finite populations of RNA viruses, but we also have no experimental evidence in favor of it. Theoretical studies and computer simulations indicate that quasispecies effects should become important when the product of effective population size and genomic mutation rate is significantly larger than one. Since for many RNA viruses the genomic mutation rate is already on the order of one $[48,49]$, even moderately large populations of RNA viruses, or populations that undergo regular bottlenecks, are candidates for quasispecies behavior.

\section{Does quasispecies theory predict an error threshold?}

The error threshold is probably one of the most misunderstood concepts of quasispecies theory. Eigen described the error threshold in his 1971 paper as a limit to the amount of information a genome can store at a given mutation rate [10]. If the mutation rate is increased beyond this limit, then the population structure breaks down, and the population disperses over sequence space.

The first important point to understand about the error threshold is that it is a deterministic effect. This means that the position of the error threshold depends only weakly on the population size, and that even in an infinite population the error threshold occurs at a finite mutation rate $[19,35]$. In this way, the error threshold differs markedly from Muller's ratchet [50], which occurs only at finite population sizes and disappears in the deterministic limit. 

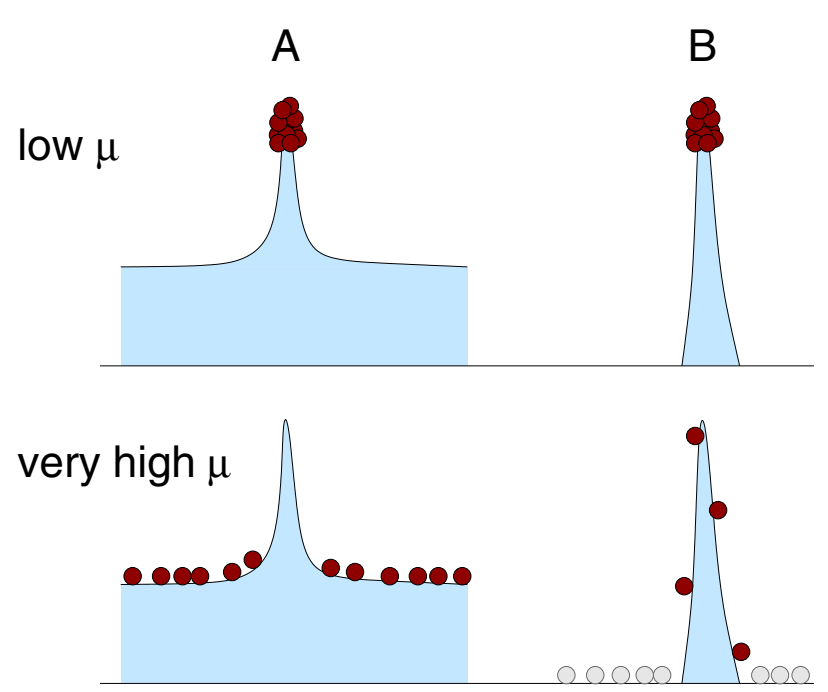

\section{Figure 2}

Schematic drawing of the error threshold. If a fitness landscape has a positive minimum fitness (case $A$ ), then at a sufficiently high mutation rate all individuals are pushed to this minimum level. The selective strength on the narrow peak is not sufficient to counteract the mutation pressure. If a fitness landscape has no minimum fitness (case B), then the mutation pressure pushes a large fraction of the population to zero fitness. The individuals with zero fitness (shown in gray) are inviable, and thus do not compete with the individuals on the fitness peak. Therefore, a few individuals will always remain on the top of the fitness peak. Note that this conclusion holds only when two assumptions are met: (i) The population is infinite. (Otherwise, stochastic effects push the population away from the peak, and we observe Muller's ratchet.) (ii) Selection is soft, that is, only relative fitness differences matter, and the overall population size is held constant at all times. (If selection is hard, then the population size will decline as the mutation rate is increased, and eventually the population can go extinct. This case is mutational meltdown.)

Second, the error threshold's existence and position depend on the choice of the fitness landscape [51-56]. Even though the error threshold is usually perceived as a general prediction of quasispecies theory, most of the literature that studies the error threshold considers only the single-peak fitness landscape, and disregards all other possible fitness functions $[10,15-21]$. The single-peak fitness landscape has the unrealistic property that all sequences have a positive replication rate, that is, there are no lethal mutants. As a result, at high mutation rates these sequences compete with the master sequence (the single sequence that has higher fitness than all other sequences), and can win this competition at sufficiently high mutation rate by sheer abundance. Can the error threshold occur in a more realistic fitness landscape that contains lethal genotypes? No. Wagner and Krall have proven mathematically that the condition for the existence of an error threshold is precisely the complete absense of lethal genotypes [53]. An intuitive explanation for this result is provided in Fig. 2.

There is certainly no lack of lethal mutants in viruses $[57,58]$, and as a consequence, viruses cannot suffer from an error threshold as defined by Eigen. If this is the case, then how can we understand the concept of lethal mutagenesis, which has recently proven successful in a variety of viruses [59-62], and for which the error threshold is generally cited as the underlying theory? The truth of the matter is that the two concepts are mostly unrelated. To understand the difference between the two, we have to understand the difference between soft and hard selection. Soft selection means that the population size is held constant, regardless of the mean fitness of the population. Under soft selection, populations cannot go extinct by definition. Since the quasispecies model is usually studied in the context of soft selection (even though it can be generalized to hard selection), the error threshold per se makes no statements about population extinction. The alternative model is hard selection, where the population size will decline if the mean fitness of the population is too low. Extinction due to mutation pressure can occur under hard selection, and is usually called mutational meltdown [63-65]. Mutational meltdown will operate in any fitness landscape, as long as the population size is sufficiently small, the mutation rate sufficiently large, or the hard selection pressure sufficiently strong. While lethal mutagenesis is probably a valid antiviral therapy, referring to it as an error-threshold related effect is at best a misnomer, and can at worst lead to poor treatment decisions brought about by a misunderstanding of the actual evolutionary dynamics that unfold under lethal mutagenesis.

Finally, it is interesting to note that in certain fitness landscapes, several error-threshold-like transitions can occur one after the other as the mutation rate increases [66]. At each transition, the population loses the ability to take advantage of a particular region of sequence space, and delocalizes over this region, while remaining localized in other regions. Tannenbaum and Shakhnovich have termed this effect the error cascade [66], and in fact, the survival-of-the-flattest effect $[32,33,46]$ can be considered as a special case of this error cascade.

\section{Conclusion}

To summarize, I have provided arguments for the following conclusions: Quasispecies theory is in perfect agreement with population genetics, it can make usefull predictions for finite populations if the product of population size and mutation rate is large, and it predicts an error threshold only for fitness landscapes that lack lethal 
mutants, and which therefore have little relevance for virus evolution.

However, these arguments do not imply that quasispecies theory is the final answer to all questions of virus evolution. Quasispecies theory has its short-comings that need to be addressed in future modeling work. Ironically, the biggest shortcoming of quasispecies theory, as far as I can see, does not have its origin in quasispecies theory being at odds with population genetics, but rather in quasispecies theory being too similar to the population genetics theory of asexual, haploid organisms. Viruses differ from other forms of life in that they don't have a well-defined ploidy. When a single virus particle infects a cell, the virus can be considered a haploid organism, and indeed the quasispecies model makes this assumption. However, frequently several virus particles coinfect the same cell, in which case the ploidy is given by the number of coinfecting particles. Standard population genetics has no model for such a variable-ploidy organism, and only a handful of authors have considered the theoretical implications of viral coinfection in detail [67-81].

\section{Authors' contributions}

COW carried out all aspects of this work.

\section{Acknowledgements}

I would like to thank Eddie Holmes, Santiago Elena, Igor Rouzine, and John Coffin for helpful comments on earlier versions of this manuscript. This work was in part supported by $\mathrm{NIH}$ grant Al 065960.

\section{References}

I. Eigen M, McCaskill J, Schuster P: Molecular Quasi-Species. J Phys Chem 1988, 92:688|-689|.

2. Eigen M, McCaskill J, Schuster P: The Molecular Quasi-Species. Adv Chem Phys 1989, 75: 149-263.

3. Domingo E, Biebricher CK, Eigen M, Holland J]: Quasispecies and RNA Virus Evolution: Principles and Consequences Georgetown, TX: Landes Bioscience; $200 \mathrm{I}$.

4. Moya A, Elena SF, Bracho A, Miralles R, Barrio E: The evolution of RNA viruses: A population genetics view. Proc Natl Acad Sci USA 2000, 97:6967-6973.

5. Jenkins GM, Worobey M, Woelk CH, Holmes EC: Evidence for the non-quasispecies evolution of RNA viruses. Mol Biol Evol 200I, 18:987-994.

6. Holmes EC, Moya A: Is the Quasispecies Concept Relevant to RNA Viruses? J Virol 2002, 76:460-462.

7. Comas I, Moya A, Gonzalez-Candelas F: Validating viral quasispecies with digital organisms: A re-examination of the critical mutation rate. BMC Evol Biol 2005, 5:5.

8. Domingo E: Quasispecies Theory in Virology. J Virol 2002, 76:463-465.

9. Eigen M: On the nature of virus quasispecies. Trends Microbiol 1996, 4:216-218

10. Eigen M: Selforganization of matter and the evolution of biological macromolecules. Die Naturwissenschaften 1971, 58:456-523.

II. Eigen M, Schuster P: The hypercycle, a principle of natural selforganization. Part A: Emergence of the hypercycle. Die Naturwissenschaften 1977, 64:54|-565.

12. Domingo E, Sabo D, Taniguchi T, Weissmann C: Nucleotide sequence heterogeneity of an RNA phage population. Cell 1978, 13:735-744.

13. Holland J, Spindler K, Horodyski F, Grabau E, Nichol S, VandePol S: Rapid evolution of RNA genomes. Science 1982, 2 I5:| 577-I 585.
14. Domingo $E$, Holland |l: RNA virus mutations and fitness for survival. Annu Rev Microbiol 1997, 51:15I-178.

15. Swetina J, Schuster P: Self-replication with errors: a model for polynucleotide replication. Biophys Chem 1982, 16:329-345.

16. Galluccio S: Exact solution of the quasispecies model in a sharply peaked fitness landscape. Phys $\operatorname{Rev} E$ 1997, 56:4526-4539.

17. Alves D, Fontanari JF: Error threshold in finite populations. Phys Rev E 1998, 57:7008-70/3.

18. Campos PRA, Fontanari JF: Finite-size scaling of the quasispecies model. Phys Rev E 1998, 58:2664-2667.

19. Campos PRA, Fontanari JF: Finite-size scaling of the error threshold transition in finite populations. J Phys A 1999 , 32:LI-L7.

20. Tannenbaum E, Deeds EJ, Shakhnovich El: Equilibrium distribution of mutators in the single fitness peak model. Phys Rev Lett 2003, 91:138105

2I. Tannenbaum E, Deeds E], Shakhnovich El: Semiconservative replication in the quasispecies model. Phys Rev E 2004, 69:061916.

22. Demetrius L: Selection and evolution in macromolecular systems. J Theor Biol 1983, 103:619-643.

23. Demetrius L, Schuster P, Sigmund K: Polynucleotide evolution and branching processes. Bull Math Biol 1985, 47:239-262.

24. Wilke CO, Ronnewinkel C, Martinetz T: Dynamic Fitness Landscapes in Molecular Evolution. Phys Rep 200I, 349:395-446.

25. Kimura M. Maruyama T: The mutational load with epistatic gene interactions in fitness. Genetics 1966, 54:1337-I35I.

26. Burger R: The Mathematical Theory of Selection, Recombination, and Mutation Chichester: Wiley; 2000.

27. van Nimwegen E, Crutchfield JP, Huynen M: Neutral Evolution of Mutational Robustness. Proc Natl Acad Sci USA 1999, 96:9716-9720.

28. Wilke CO, Adami C: Evolution of mutational robustness. Mut Res 2003, 522:3-II.

29. Thompson CJ, McBride JL: On Eigen's Theory of Self-Organization of Matter and the Evolution of Biological Macromolecules. Math Biosci 1974, 21:127-142.

30. Jones BL, Enns RH, Rangnekar SS: On the theory of selection of coupled macromolecular systems. Bull Math Biol 1976, 38: $15-28$.

31. Moran PAP: Global stability of genetic systems governed by mutation and selection. Math Proc Camb Phil Soc 1976, 80:331-336.

32. Schuster P, Swetina J: Stationary mutant distributions and evolutionary optimization. Bull Math Biol 1988, 50:635-660.

33. Wilke CO, Wang JL, Ofria C, Lenski RE, Adami C: Evolution of digital organisms at high mutation rate leads to survival of the flattest. Nature 200I, 4 | 2:33|-333.

34. McCaskill JS: A stochastic theory of macromolecular evolution. Biological Cybernetics 1984, 50:63-73.

35. Nowak M, Schuster P: Error Thresholds of Replication in Finite Populations-Mutation Frequencies and the Onset of Muller's Ratchet. I Theor Biol 1989, 1 37:375-395.

36. Woodcock G, Higgs PG: Population evolution on a multiplicative single-peak fitness landscape. J Theor Biol 1996, 179:61-73.

37. van Nimwegen E, Crutchfield IP, Mitchell M: Statistical Dynamics of the Royal Road Genetic Algorithm. Theoretical Computer Science 1999, 229:4I-102.

38. Rouzine IM, Wakeley J, Coffin JM: The solitary wave of asexual evolution. Proc Natl Acad Sci USA 2003, 100:587-592.

39. Bornberg-Bauer E, Chan HS: Modeling evolutionary landscapes: Mutational stability, topology, and superfunnels in sequence space. Proc Natl Acad Sci USA 1999, 96: 10689- 10694.

40. Wilke CO: Adaptive Evolution on Neutral Networks. Bull Math Biol 200I, 63:7I5-730.

41. Wilke CO: Molecular clock in neutral protein evolution. $B M C$ Genetics 2004, 5:25.

42. Iwasa $Y$, Michor F, Nowak MA: Evolutionary dynamics of invasion and escape. J Theor Biol 2003, 226:205-2I4.

43. Wilke CO: Probability of Fixation of an Advantageous Mutant in a Viral Quasispecies. Genetics 2003, 163:467-474.

44. Barton NH: Linkage and the limits to natural selection. Genetics 1995, |40:82|-84|.

45. Johnson T, Barton NH: The Effect of Deleterious Alleles on Adaptation in Asexual Populations. Genetics 2002, 162:395-4|I. 
46. Wilke CO: Selection for fitness versus selection for robustness in RNA secondary structure folding. Evolution 200I, 55:24I2-2420

47. Price DA, Goulder PJR, Klenerman P, Sewell AK, Easterbrook PJ, Troop M, Bangham CRM, Phillips RE: Positive selection of HIV-I cytotoxic $T$ lymphocyte escape variants during primary infection. Proc Natl Acad Sci USA 1997, 94:1890-1895.

48. Drake JW: Rates of spontaneous mutation among RNA viruses. Proc Natl Acad Sci USA 1993, 90:4I7I-4I75.

49. Drake JW, Holland J]: Mutation rates among RNA viruses. Proc Natl Acad Sci USA 1999, 96:13910-13913.

50. Haigh J: Accumulation of deleterious mutations: Muller's ratchet. Theor Popul Biol 1978, 14:25I-267.

51. Maynard Smith J: Models of evolution. Proc R Soc London B 1983 , 219:315-325.

52. Charlesworth B: Mutation-selection balance and the evolutionary advantage of sex and recombination. Genet Res 1990 55:199-22I.

53. Wagner GP, Krall P: What is the difference between models of error thresholds and Muller's ratchet? J Math Biol 1993, 32:33-44.

54. Higgs PG: Error thresholds and stationary mutant distributions in multi-locus diploid genetics models. Genet Res 1994 63:63-78.

55. Wiehe T: Model dependency of error thresholds: the role of fitness functions and contrasts between the finite and infinite sites models. Genet Res 1997, 69:127-136.

56. Baake E, Wagner H: Mutation-selection models solved exactly with methods of statistical mechanic. Genet Res 2001, 78:93-117.

57. Malpica JM, Fraile A, Moreno I, Obies C, Drake JW, García-Arenal F: The Rate and Character of Spontaneous Mutation in an RNA Virus. Genetics 2002, 162:1505-151I.

58. Sanjuán R, Moya A, Elena SF: The distribution of fitness effects caused by single-nucleotide substitutions in an RNA virus. Proc Natl Acad Sci USA 2004, 10 1:8396-840I.

59. Pariente N, Sierra S, Lowenstein PR, Domingo E: Efficient virus extinction by combinations of a mutagen and antiviral inhibitors. J Virol 200I, 75:9723-9730.

60. Crotty S, Cameron CE, Andino R: RNA virus error catastrophe: Direct molecular test by using ribavirin. Proc Natl Acad Sci USA 200I, 98:6895-6900.

61. Grande-Perez A, Sierra S, Castro MG, Domingo E, Lowenstein PR: Molecular indetermination in the transition to error catastrophe: Systematic elimination of lymphocytic choriomeningitis virus through mutagenesis does not correlate linearly with large increases in mutant spectrum complexity. Proc Natl Acad Sci USA 2002, 99: I2938-12943.

62. Anderson JP, Daifuku R, Loeb LA: Viral error catastrophe by mutagenic nucleosides. Annu Rev Microbiol 2004, 58: 183-205.

63. Gabriel W, Lynch M, Burger R: Muller's ratchet and mutational meltdowns. Evolution 1993, 47:1744-1757.

64. Lynch M, Burger R, Butcher D, Gabriel W: The mutational meltdown in asexual populations. J Hered 1993, 84:339-344.

65. Lynch $M$, Conery J, Burger R: Mutation accumulation and the extinction of small populations. Am Nat 1995, 146:489-5I8.

66. Tannenbaum E, Shakhnovich El: Solution of the quasispecies model for an arbitrary gene network. Phys Rev E 2004, 70:021903.

67. Nee S, Maynard Smith J: The evolutionary biology of molecular parasites. Parasitology 1990, 100:S5-SI8.

68. Bangham CRM, Kirkwood TBL: Defective interfering particles: effects in modulating virus growth and persistence. Virology 1990, I 79:821-826.

69. Kirkwood TBL, Bangham CRM: Cycles, chaos, and evolution in virus cultures: $A$ model of defective interfering particles. Proc Natl Acad Sci USA 1994, 91 :8685-8689.

70. Szathmáry E: Natural selection and dynamical coexistence of defective and complementing virus segments. J theor Biol 1992, I 57:383-406.

71. Szathmáry E: Co-operation and defection: playing the field in virus dynamics. J theor Biol 1993, 165:34I-356

72. Turner PE, Burch CL, Hanley KA, Chao L: Hybrid frequencies confirm limit to coinfection in the RNA bacteriophage $\Phi 6$. J Virol 1999, 73:2420-2424
73. Turner PE, Chao L: Prisoner's dilemma in an RNA virus. Nature 1999, 398:44|-443.

74. Brown SP: Collective action in an RNA virus. J Evol Biol 200I, I 4:82I-828.

75. Frank SA: Within-host spatial dynamics of viruses and defective interfering particles. J theor Biol 2000, 206:279-290.

76. Frank SA: Multiplicity of infection and the evolution of hybrid incompatibility in segmented viruses. Heredity 200I, 87:522-529.

77. Wilke CO, Novella IS: Phenotypic mixing and hiding may contribute to memory in viral quasispecies. BMC Microbiology 2003 , 3:11.

78. Novella IS, Reissig DD, Wilke CO: Density-dependent selection in vesicular stomatitis virus. J Virol 2004, 78:5799-5804.

79. Wilke CO, Reissig DD, Novella IS: Replication at periodically changing multiplicity of infection promotes stable coexistence of competing viral populations. Evolution 2004, 58:900-905.

80. Froissart R, Wilke CO, Montville R, Remold SK, Chao L, Turner PE: Co-infection weakens selection against epistatic mutations in RNA viruses. Genetics 2004, 168:9-19.

8I. Bretscher MT, Althaus CL, Müller V, Bonhoeffer S: Recombination in HIV and the evolution of drug resistance: for better or for worse? BioEssays 2004, 26: 180-188.
Publish with Bio Med Central and every scientist can read your work free of charge

"BioMed Central will be the most significant development for disseminating the results of biomedical research in our lifetime. "

Sir Paul Nurse, Cancer Research UK

Your research papers will be:

- available free of charge to the entire biomedical community

- peer reviewed and published immediately upon acceptance

- cited in PubMed and archived on PubMed Central

- yours - you keep the copyright
BiolMedcentral 